Vietnam Journal of Mechanics, VAST, Vol.38, No. 2 (2016), pp. $103-122$

DOI:10.15625/0866-7136/38/2/6730

\title{
FREE VIBRATION OF FUNCTIONALLY GRADED SANDWICH PLATES WITH STIFFENERS BASED ON THE THIRD-ORDER SHEAR DEFORMATION THEORY
}

\author{
Pham Tien Dat, Do Van Thom*, Doan Trac Luat \\ Le Quy Don Technical University, Hanoi, Vietnam \\ *E-mail: promotion6699@gmail.com \\ Received August 11, 2015
}

\begin{abstract}
In this paper, the free vibration of functionally sandwich grades plates with stiffeners is investigated by using the finite element method. The material properties are assumed to be graded in the thickness direction by a power-law distribution. Based on the third-order shear deformation theory, the governing equations of motion are derived from the Hamilton's principle. A parametric study is carried out to highlight the effect of material distribution, stiffener parameters on the free vibration characteristics of the plates.

Keywords: Stiffened plate, sandwich, vibration, functionally graded materials.
\end{abstract}

\section{INTRODUCTION}

Functionally graded materials (FGMs) made of two constituents, mainly metal and ceramic are widely used. The composition is varied continuously along certain directions according to volume fration from a ceramic-rich surface to a metal-rich surface. Many researchers in past have worked upon to understand behavior of functionally graded plates. Reddy [1] carried out nonliear finite element static and dynamic analyses of functionally graded plates using Navier's solution, Vel and Batra [2] presented threedimendional exact solutions for free and forced vibrations of simply supported functionally graded rectangular plates, Tinh Quoc Bui et al [3] used finite element method to study static bending and vibration of heated FG plates. Dao Huy Bich et al [4,5], Nguyen Dinh Duc et al $[6,7]$ used analytical method to study vibration, nonlinear respones of eccentrically stiffened functionally graded cylindrical panels $[4,5]$ and nonlinear postbucking of FGM plate and eccentrically stiffened thin FGM resting on elastic foundations in thermal environments [6,7]. Several studies have been performed to analyze the behaviour of FG sandwich structures, Zenkour [8], Alipour and Shariyat [9].

In this paper, the free vibartion of stiffened FG sandwich plates is sudied by using finite element method. Here we present FG sandwich plate with material properties

(c) 2016 Vietnam Academy of Science and Technology 
symmetric about the mid-plane. The faces of the plate consist of a FGM with properties varying only in the thickness direction. Such faces can be made by mixing two different material phases, for example, a metal and a ceramic. The core material may be homogeneous and can be made by one of these materials, for example, a ceramic or a metal. Eigenfrequencies and mode shapes of stiffened FG sandwich plates are presented using the higher-order shear deformation theory.

\section{GOVERNING EQUATIONS AND FINITE ELEMENT ELEMENT FORMULATION}

Consider a stiffened FG sandwich plate with thickness, long, wide of the plate are $2 h, a, b$, and depth, width of the stiffener are $h_{s}, b_{s}$, respectively (Fig. 1 ). The $x y$-plane is the mid-plane of the plate, and the positive $z$-axis is upward from the mid-plane. The power law distribution is used for describing the volume fraction of the ceramic $\left(V_{c}^{(i)}\right)$ and the metal $\left(V_{m}^{(i)}\right)$ in $i$-th layer $(i=1,2,3)$ as follow

$$
V_{m}^{(i)}+V_{c}^{(i)}=1 \text {. }
$$
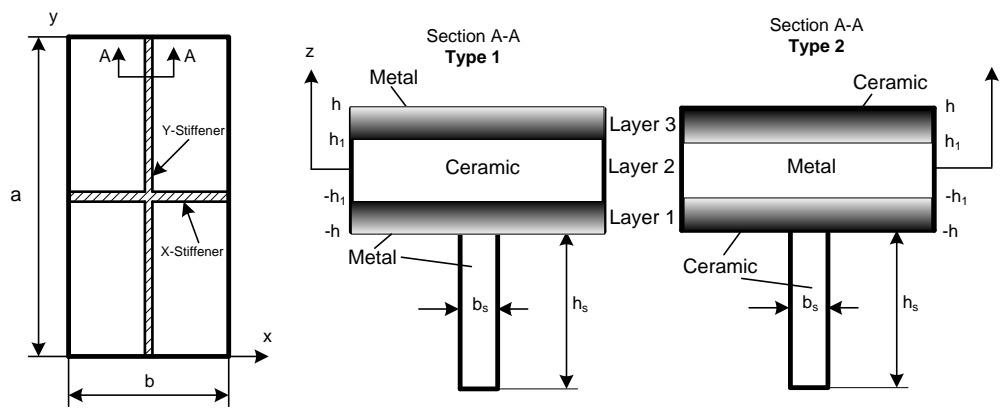

Fig. 1. A FG plate stiffened by an $x$-direction and an $y$-direction stiffeners

For type 1

For type 2

$$
\begin{cases}V_{c}^{(1)}=\left(\frac{z+h}{h-h_{1}}\right)^{n} ; & -h \leq z \leq-h_{1} \\ V_{c}^{(2)}=1 \quad & -h_{1} \leq z \leq h \\ V_{c}^{(3)}=\left(\frac{z-h}{h_{1}-h}\right)^{n} ; & h_{1} \leq z \leq h\end{cases}
$$

$$
\begin{cases}V_{c}^{(1)}=\left(\frac{z+h_{1}}{h_{1}-h}\right)^{n} ; & -h \leq z \leq-h_{1} \\ V_{c}^{(2)}=0 & ; \quad-h_{1} \leq z \leq h \\ V_{c}^{(3)}=\left(\frac{z-h_{1}}{h-h_{1}}\right)^{n^{\prime}} ; & h_{1} \leq z \leq h\end{cases}
$$


Where, $2 h$-thickness of the plate, $2 h_{1}$-thickness of second layer; $n$-the gradient in$\operatorname{dex}(n \geq 0)$; $z$-the thickness coordinate variable, and subscripts $c$ and $m$ represent the ceramic and metal constituents, respectively.

In this study, the material properties, i.e., Young's modulus $E$, Poisson's ratio $v$, and the mass density $\rho$, can be expressed by the rule of mixture as [1]

$$
P^{(i)}(z)=P_{m}+\left(P_{c}-P_{m}\right) V_{c}^{(i)},
$$

where $P^{(i)}$ is the material property of $i$-th layer.

In [10] the three-dimensional displacement field $(u, v, w)$ can be expressed in terms of nine unknown variables as follows

$$
\begin{aligned}
& u(x, y, z)=u^{0}(x, y)+z \cdot \psi_{x}(x, y)+z^{2} \cdot \xi_{x}(x, y)+z^{3} \cdot \Phi_{x}(x, y), \\
& v(x, y, z)=v^{0}(x, y)+z \cdot \psi_{y}(x, y)+z^{2} \cdot \xi_{y}(x, y)+z^{3} \cdot \Phi_{y}(x, y), \\
& w(x, y, z)=w_{0}(x, y),
\end{aligned}
$$

where $u^{0}, v^{0}, w^{0}$ represent the displacements at the mid-plane of the plate in the $x, y$ and $z$ directions, respectively; $\psi_{x}, \psi_{y}$ denote the transverse normal rotations of the $y$ and $x$ axes; $\xi_{x}, \xi_{y}$ denote the higher order displacements and $\Phi_{x}, \Phi_{y}$ denote the higher order transverse rotations.

\subsection{Plate element}

We consider a plate element with 4 nodes, each node has 9 degrees of freedom (see Fig. 2).

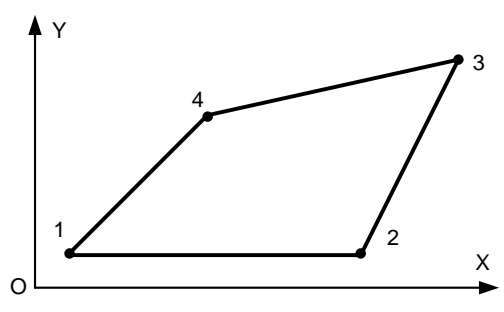

(a) Plate element in Decaster system



(b) Natural coordinate system

Fig. 2. Rectangular isoparametric plate element

For the plate element, displacement vector of $i$-th node has the form

$$
\left\{q_{i}\right\}=\left\{u_{i}, v_{i}, w_{i}, \psi_{x i}, \psi_{y i}, \xi_{x i}, \xi_{y i}, \Phi_{x i}, \Phi_{y i}\right\}^{T} ; \quad i=\overline{1,4}
$$

Displacement vector at any point of the plate element $\{u\}$ is computed using shape function $N_{i}$ as follows

$$
\{u\}=\sum_{i=1}^{4} N_{i} \cdot\left\{q_{i}\right\},
$$




$$
\begin{array}{ll}
N_{1}=0,25(1-r)(1-s), & N_{2}=0,25(1+r)(1-s), \\
N_{3}=0,25(1+r)(1+s), & N_{4}=0,25(1-r)(1+s) .
\end{array}
$$

The strain vector can be expressed in the form

$$
\{\varepsilon\}=\left\{\varepsilon^{0}\right\}+z\{\kappa\}+z^{2}\{\chi\}+z^{3}\{\eta\}, \quad\{\gamma\}=\left\{\gamma^{0}\right\}+z\left\{\kappa^{\prime}\right\}+z^{2}\left\{\chi^{\prime}\right\},
$$

where

$$
\begin{gathered}
\left\{\varepsilon^{0}\right\}=L_{1}\{u\}=L_{1} \sum_{i=1}^{4} N_{i}\left\{q_{i}\right\}=\left[B_{1}\right]\{q\}_{e}, \\
\{\kappa\}=L_{2}\{u\}=L_{2} \sum_{i=1}^{4} N_{i}\left\{q_{i}\right\}=\left[B_{2}\right]\{q\}_{e},\{\chi\}=L_{3}\{u\}=L_{3} \sum_{i=1}^{4} N_{i}\left\{q_{i}\right\}=\left[B_{3}\right]\{q\}_{e}, \\
\{\eta\}=L_{4}\{u\}=L_{4} \sum_{i=1}^{4} N_{i}\left\{q_{i}\right\}=\left[B_{4}\right]\{q\}_{e},\left\{\gamma^{0}\right\}=L_{1}^{\prime}\{u\}=L_{1}^{\prime} \sum_{i=1}^{4} N_{i}\left\{q_{i}\right\}=\left[B_{1}^{\prime}\right]\{q\}_{e}, \\
\left\{\kappa^{\prime}\right\}=L_{2}^{\prime}\{u\}=L_{2}^{\prime} \sum_{i=1}^{4} N_{i}\left\{q_{i}\right\}=\left[B_{2}^{\prime}\right]\{q\}_{e},\left\{\chi^{\prime}\right\}=L_{3}^{\prime}\{u\}=L_{3}^{\prime} \sum_{i=1}^{4} N_{i}\left\{q_{i}\right\}=\left[B_{3}^{\prime}\right]\{q\}_{e},
\end{gathered}
$$

where

$$
\left[B_{j}\right]=L_{j} \sum_{i=1}^{4} N_{i}, \quad\left[B_{j}^{\prime}\right]=L_{j}^{\prime} \sum_{i=1}^{4} N_{i},
$$

and $\{q\}_{e}=\left\{\left\{q_{1}\right\}, \ldots,\left\{q_{4}\right\}\right\}^{T}$ - displacement vector of plate element. $L_{j}, L_{j}{ }^{\prime}$ - standard strain-displacement matrix of plate element.

The stress vector at any point in $i$-th layer of the plate is expressed as

$$
\left\{\begin{array}{l}
\{\sigma\}^{(i)} \\
\{\tau\}^{(i)}
\end{array}\right\}=\left[\begin{array}{cc}
{\left[D_{m i}\right]} & {[0]} \\
{[0]} & {\left[D_{s i}\right]}
\end{array}\right]\left\{\begin{array}{l}
\{\varepsilon\}^{(i)} \\
\{\gamma\}^{(i)}
\end{array}\right\},
$$

where

$$
\begin{gathered}
\{\sigma\}^{(i)}=\left\{\sigma_{x}^{(i)} \sigma_{y}^{(i)} \tau_{x y}^{(i)}\right\}^{T}, \quad\{\tau\}^{(i)}=\left\{\tau_{x z}^{(i)} \tau_{y z}^{(i)}\right\}, \\
{\left[D_{m i}\right]=\frac{E^{(i)}(z)}{1-\left(v^{(i)}\right)^{2}}\left[\begin{array}{ccc}
1 & v^{(i)} & 0 \\
v^{(i)} & 1 & 0 \\
0 & 0 & \frac{1-v^{(i)}}{2}
\end{array}\right], \quad\left[D_{s i}\right]=\frac{E^{(i)}(z)}{2\left(1+v^{(i)}\right)} \operatorname{diag}(2,2) .}
\end{gathered}
$$

\section{2. $x$-Stiffener element}

The displacement field of $x$-Stiffened can be expressed as (see Fig. 3)

$$
\begin{aligned}
& u_{x s}(x, y, z)=u_{x s}^{0}(x, y)+z \psi_{x s}(x, y)+z^{2} \xi_{x s}(x, y)+z^{3} \Phi_{x s}(x, y), \\
& v_{x s}(x, y, z)=0, \\
& w_{x s}(x, y, z)=w_{x s}^{0}(x, y) .
\end{aligned}
$$

The strain vector for the $x$-stiffener element has the form

$$
\{\varepsilon\}_{x S}=\left\{\varepsilon^{0}\right\}_{x s}+z\{\kappa\}_{x s}+z^{2}\{\chi\}_{x S}+z^{3}\{\eta\}_{x S},\{\gamma\}_{x S}=\left\{\gamma^{0}\right\}_{x s}+z\left\{\kappa^{\prime}\right\}_{x s}+z^{2}\left\{\chi^{\prime}\right\}_{x s} .
$$




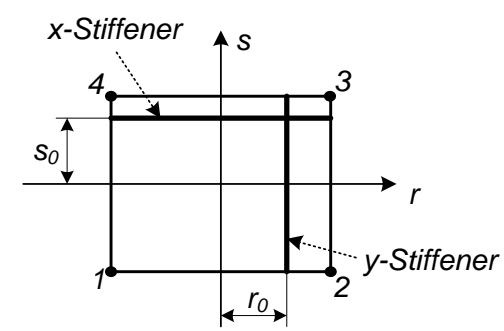

Fig. 3. Plate element and stiffener element

where

$$
\begin{gathered}
\left\{\varepsilon^{0}\right\}_{x s}=L_{1 x s}\{u\}_{x s}=\left[B_{1 x s}\right]\{q\}_{\text {exs }}, \\
\{\kappa\}_{x s}=L_{2 x s}\{u\}_{x s}=\left[B_{2 x s}\right]\{q\}_{e x s}, \quad\{\chi\}_{x s}=L_{3 x s}\{u\}_{x s}=\left[B_{3 x s}\right]\{q\}_{e x s}, \\
\{\eta\}_{x s}=L_{4 x s}\{u\}_{x s}=\left[B_{4 x s}\right]\{q\}_{e x s}, \quad\left\{\gamma^{0}\right\}_{x s}=L_{1 x s}^{\prime}\{u\}_{x s}=\left[B_{1 x s}^{\prime}\right]\{q\}_{e x s}, \\
\left\{\kappa^{\prime}\right\}_{x s}=L_{2 x s}^{\prime}\{u\}_{x s}=\left[B_{2 x s}^{\prime}\right]\{q\}_{\text {exs }}, \quad\left\{\chi^{\prime}\right\}_{x s}=L_{3 x s}^{\prime}\{u\}_{x s}=\left[B_{3 x s}^{\prime}\right]\{q\}_{\text {exs }},
\end{gathered}
$$

where $\left[B_{j x s}\right]=L_{j x s} \sum_{i=1}^{4} N_{i x s} ;\left[B_{j x s}^{\prime}\right]=L_{j x s}^{\prime} \sum_{i=1}^{4} N_{i x s} ; N_{i x s}$ - the shape functions of $x$-stiffener which can be obtained by substituting $s=s_{0}$ into Eq. (8); $\{q\}_{\text {exs }}$ - displacement vector of $x$-stiffener coordinate system; $L_{j x s}, L_{j x s}^{\prime}$ - standard strain-displacement matrices of $x$ stiffener element.

The stress vector at any point in the stiffener is expressed as

$$
\left\{\begin{array}{l}
\{\sigma\}_{x s} \\
\{\tau\}_{x s}
\end{array}\right\}=\left[\begin{array}{cc}
{\left[D_{m x s}\right]} & {[0]} \\
{[0]} & {\left[D_{s x s}\right]}
\end{array}\right]\left\{\begin{array}{l}
\{\varepsilon\}_{x s} \\
\{\gamma\}_{x s}
\end{array}\right\}
$$

where

$$
\begin{gathered}
\{\sigma\}_{x s}=\left\{\sigma_{x} \sigma_{y} \tau_{x y}\right\}_{x s}^{T}, \quad\{\tau\}_{x s}=\left\{\tau_{x z} \tau_{y z}\right\}_{x s}^{T}, \\
{\left[D_{m x s}\right]=\frac{E_{x s}}{1-v_{x s}^{2}}\left[\begin{array}{ccc}
1 & v_{x s} & 0 \\
v_{x s} & 1 & 0 \\
0 & 0 & \frac{1-v_{x s}}{2}
\end{array}\right], \quad\left[D_{s x s}\right]=\frac{E_{x s}}{2\left(1+v_{x s}\right)} \operatorname{diag}(2,2) .}
\end{gathered}
$$

\section{3. y-Stiffener element}

The displacement field of $y$-stiffened has the form

$$
\begin{aligned}
& u_{y s}(x, y, z)=0 \\
& v_{y s}(x, y, z)=v_{y s}^{0}(x, y)+z \psi_{y s}(x, y)+z^{2} \xi_{y s}(x, y)+z^{3} \Phi_{y s}(x, y), \\
& w_{y s}(x, y, z)=w_{y s}^{0}(x, y) .
\end{aligned}
$$

The strain vector for the $y$-stiffener element is expressed in the form

$$
\{\varepsilon\}_{y s}=\left\{\varepsilon^{0}\right\}_{y s}+z\{\kappa\}_{y s}+z^{2}\{\chi\}_{y s}+z^{3}\{\eta\}_{y s},\{\gamma\}_{y s}=\left\{\gamma^{0}\right\}_{y s}+z\left\{\kappa^{\prime}\right\}_{y s}+z^{2}\left\{\chi^{\prime}\right\}_{y s} ，
$$


where

$$
\begin{aligned}
& \left\{\varepsilon^{0}\right\}_{y s}=L_{1 y s}\{u\}_{y s}=\left[B_{1 y s}\right]\{q\}_{e y s}, \\
& \{\kappa\}_{y s}=L_{2 y s}\{u\}_{y s}=\left[B_{2 y s}\right]\{q\}_{e y s}, \quad\{\chi\}_{y s}=L_{3 y s}\{u\}_{y s}=\left[B_{3 y s}\right]\{q\}_{e y s}, \\
& \{\eta\}_{y s}=L_{4 y s}\{u\}_{y s}=\left[B_{4 y s}\right]\{q\}_{e y s}, \quad\left\{\gamma^{0}\right\}_{y s}=L_{1 y s}^{\prime}\{u\}_{y s}=\left[B_{1 y s}^{\prime}\right]\{q\}_{e y s}, \\
& \left\{\mathcal{K}^{\prime}\right\}_{y s}=L_{2 y s}^{\prime}\{u\}_{y s}=\left[B_{2 y s}^{\prime}\right]\{q\}_{e y s}, \quad\left\{\chi^{\prime}\right\}_{y s}=L_{3 y s}^{\prime}\{u\}_{y s}=\left[B_{3 y s}^{\prime}\right]\{q\}_{e y s},
\end{aligned}
$$

where $\left[B_{j y s}\right]=L_{j y s} \sum_{i=1}^{4} N_{i y s} ;\left[B_{j y s}^{\prime}\right]=L_{j y s}^{\prime} \sum_{i=1}^{4} N_{i y s} ; N_{i y s}$ - shape functions of $y$-stiffener which can be obtained by substituting $r=r_{0}$ into Eq. (8); $\{q\}_{\text {eys }}$ - displacement vector of $y$ stiffener coordinate system; $L_{j y s}, L_{j y s}^{\prime}$ - standard strain-displacement matrix of $y$-stiffener element.

The stress vector at any point in the stiffener is expressed as

$$
\left\{\begin{array}{l}
\{\sigma\}_{y s} \\
\{\tau\}_{y s}
\end{array}\right\}=\left[\begin{array}{cc}
{\left[D_{m y s}\right]} & {[0]} \\
{[0]} & {\left[D_{s y s}\right]}
\end{array}\right]\left\{\begin{array}{l}
\{\varepsilon\}_{y s} \\
\{\gamma\}_{y s}
\end{array}\right\},
$$

where

$$
\begin{aligned}
& \{\sigma\}_{y s}=\left\{\begin{array}{lll}
\sigma_{x} & \sigma_{y} & \tau_{x y}
\end{array}\right\}_{y s}^{T}, \quad\{\tau\}_{y s}=\left\{\begin{array}{ll}
\tau_{x z} & \tau_{y z}
\end{array}\right\}_{y s}^{T}, \\
& {\left[D_{m y s}\right]=\frac{E_{y s}}{1-v_{y s}^{2}}\left[\begin{array}{ccc}
1 & v & 0 \\
v & 1 & 0 \\
0 & 0 & \frac{1-v_{y s}}{2}
\end{array}\right], \quad\left[D_{s y s}\right]=\frac{E_{y s}}{2\left(1+v_{y s}\right)} \operatorname{diag}(2,2) .}
\end{aligned}
$$

\subsection{Transformation matrices}

We consider that the $x$-Stiffener is attached to the lower side of the plate, the conditions of displacement compatibility along the line of connection can be written as

$$
\{u\}_{z=-0.5 h}=\{u\}_{x \mid z=0.5 h_{s x}} .
$$

Using Eqs. (5) and (18), the conditions in Eq. (36) thus lead to

$$
\begin{aligned}
& {\left[u_{x}^{0}\right]_{i}=\left[u^{0}\right]_{i}+e_{x}^{1}\left[\psi_{x}\right]_{i}+e_{x}^{2}\left[\xi_{x}\right]_{i}+e_{x}^{3}\left[\Phi_{x}\right]_{i},} \\
& {\left[\psi_{x}\right]_{i}=\left[\psi_{x s}\right]_{i}} \\
& {\left[\xi_{x}\right]_{i}=\left[\tilde{\zeta}_{x s}\right]_{i}} \\
& {\left[\Phi_{x}\right]_{i}=\left[\Phi_{x s}\right]_{i}}
\end{aligned}
$$

where

$$
e_{x}^{1}=-\left(h+h_{x s}\right) / 2, \quad e_{x}^{2}=\left(h^{2}-h_{x s}^{2}\right) / 4, \quad e_{x}^{3}=-\left(h^{3}+h_{x s}^{3}\right) / 8
$$


Expressing in matrix form

$$
\left\{\begin{array}{c}
u_{x}^{0} \\
0 \\
w_{x} \\
\psi_{x-x} \\
0 \\
\xi_{x-x} \\
0 \\
\Phi_{x-x} \\
0
\end{array}\right\}_{i}\left[\begin{array}{ccccccccc}
1 & 0 & 0 & e_{x}^{1} & 0 & e_{x}^{2} & e_{x}^{1} & 0 & e_{x}^{2} \\
0 & 0 & 0 & 0 & 0 & 0 & 0 & 0 & 0 \\
0 & 0 & 1 & 0 & 0 & 0 & 0 & 0 & 0 \\
0 & 0 & 0 & 1 & 0 & 0 & 0 & 0 & 0 \\
0 & 0 & 0 & 0 & 0 & 0 & 0 & 0 & 0 \\
0 & 0 & 0 & 0 & 0 & 1 & 0 & 0 & 0 \\
0 & 0 & 0 & 0 & 0 & 0 & 0 & 0 & 0 \\
0 & 0 & 0 & 0 & 0 & 0 & 0 & 1 & 0 \\
0 & 0 & 0 & 0 & 0 & 0 & 0 & 0 & 0
\end{array}\right]\left\{\begin{array}{l}
u^{0} \\
v \\
w_{0} \\
\psi_{x} \\
\psi_{y} \\
\xi_{x} \\
\xi_{y} \\
\Phi_{x} \\
\Phi_{y}
\end{array}\right\}_{i} i=1,4
$$

or

$$
\{u\}_{x i}=T_{x s}\{u\}_{i} .
$$

The nodal displacement vector can be written in the form

$$
\{q\}_{e x}=T_{x}\{q\}_{e}
$$

where

$$
T_{x}=T_{x s} \cdot \operatorname{diag}(4,4) \text {. }
$$

The transformation matrix for the $y$-stiffener element can be derived in a similar way in the form

$$
\{q\}_{y e}=T_{y}\{q\}_{e} .
$$

\subsection{Weak form of the stiffened plate problem}

The elastic strain energy of the plate is written as [11]

$$
U=\frac{1}{2} \sum_{N_{p}} \int_{V_{e}}\{\varepsilon\}^{T}\{\sigma\} d V_{e}+\frac{1}{2} \sum_{N_{x s} V_{e x}}\{\varepsilon\}_{x}^{T}\{\sigma\}_{x} d V_{e x}+\frac{1}{2} \sum_{N_{y s} V_{V_{e y}}}\{\varepsilon\}_{y}^{T}\{\sigma\}_{y} d V_{e y},
$$

or in the matrix form

$$
U=\frac{1}{2} \sum_{N_{p}}\{q\}_{e}^{T}[K]_{e}\{q\}_{e}+\frac{1}{2} \sum_{N_{x s}}\{q\}_{e x}^{T}[K]_{e x}\{q\}_{e x}^{T}+\frac{1}{2} \sum_{N_{y s}}\{q\}_{e y}^{T}[K]_{e y}\{q\}_{e y}^{T},
$$

where

$$
\left.[K]_{e}=\int \begin{array}{l}
{\left[B_{1}\right]^{T}[A]\left[B_{1}\right]+\left[B_{1}\right]^{T}[B]\left[B_{2}\right]+\left[B_{1}\right]^{T}[D]\left[B_{3}\right]+\left[B_{1}\right]^{T}[E]\left[B_{4}\right]+\left[B_{2}\right]^{T}[B]\left[B_{1}\right]} \\
+\left[B_{2}\right]^{T}[D]\left[B_{2}\right]+\left[B_{2}\right]^{T}[E]\left[B_{3}\right]+\left[B_{2}\right]^{T}[F]\left[B_{4}\right]+\left[B_{3}\right]^{T}[D]\left[B_{1}\right]+\left[B_{3}\right]^{T}[E]\left[B_{2}\right] \\
+\left[B_{3}\right]^{T}[F]\left[B_{3}\right]+\left[B_{3}\right]^{T}[G]\left[B_{4}\right]+\left[B_{4}\right]^{T}[E]\left[B_{1}\right]+\left[B_{4}\right]^{T}[F]\left[B_{2}\right]+ \\
\left.+\left[B_{4}\right]^{T}[G]\left[B_{3}\right]+\left[B_{4}\right]^{T}[H]\left[B_{4}\right]+\left[B_{1}\right]^{\prime}\right]^{T}\left[A^{\prime}\right]\left[B_{1}^{\prime}\right]+\left[B_{1}^{\prime}\right]^{T}\left[B^{\prime}\right]\left[B_{2}^{\prime}\right]+ \\
+\left[B_{1}^{\prime}\right]^{T}\left[D^{\prime}\right]\left[B_{3}^{\prime}\right]+\left[B_{2}^{\prime}\right]^{T}\left[B^{\prime}\right]\left[B_{1}^{\prime}\right]+\left[B_{2}^{\prime}\right]^{T}\left[D^{\prime}\right]\left[B_{2}^{\prime}\right]+\left[B_{2}^{\prime}\right]^{T}\left[E^{\prime}\right]\left[B_{3}{ }^{\prime}\right]+ \\
+\left[B_{3}^{\prime}\right]^{T}\left[D^{\prime}\right]\left[B_{1}^{\prime}\right]+\left[B_{3}^{\prime}\right]^{T}\left[E^{\prime}\right]\left[B_{2}^{\prime}\right]+\left[B_{3}^{\prime}\right]^{T}\left[F^{\prime}\right]\left[B_{3}^{\prime}\right]
\end{array}\right] d S_{e}=
$$




$$
\begin{aligned}
& =\sum_{i=1}^{2} \sum_{j=1}^{2}\left[\begin{array}{l}
{\left[B_{1}\right]^{T}[A]\left[B_{1}\right]+\left[B_{1}\right]^{T}[B]\left[B_{2}\right]+\left[B_{1}\right]^{T}[D]\left[B_{3}\right]+\left[B_{1}\right]^{T}[E]\left[B_{4}\right]+\left[B_{2}\right]^{T}[B]\left[B_{1}\right]} \\
+\left[B_{2}\right]^{T}[D]\left[B_{2}\right]+\left[B_{2}\right]^{T}[E]\left[B_{3}\right]+\left[B_{2}\right]^{T}[F]\left[B_{4}\right]+\left[B_{3}\right]^{T}[D]\left[B_{1}\right]+\left[B_{3}\right]^{T}[E]\left[B_{2}\right] \\
+\left[B_{3}\right]^{T}[F]\left[B_{3}\right]+\left[B_{3}\right]^{T}[G]\left[B_{4}\right]+\left[B_{4}\right]^{T}[E]\left[B_{1}\right]+\left[B_{4}\right]^{T}[F]\left[B_{2}\right]+ \\
+\left[B_{4}\right]^{T}[G]\left[B_{3}\right]+\left[B_{4}\right]^{T}[H]\left[B_{4}\right]+\left[B_{1}^{\prime}\right]^{T}\left[A^{\prime}\right]\left[B_{1}^{\prime}\right]+\left[B_{1}^{\prime}\right]^{T}\left[B^{\prime}\right]\left[B_{2}^{\prime}\right]+ \\
+\left[B_{1}^{\prime}\right]^{T}\left[D^{\prime}\right]\left[B_{3}^{\prime}\right]+\left[B_{2}^{\prime}\right]^{T}\left[B^{\prime}\right]\left[B_{1}^{\prime}\right]+\left[B_{2}^{\prime}\right]^{T}\left[D^{\prime}\right]\left[B_{2}^{\prime}\right]+\left[B_{2}^{\prime}\right]^{T}\left[E^{\prime}\right]\left[B_{3}^{\prime}\right]+ \\
+\left[B_{3}^{\prime}\right]^{T}\left[D^{\prime}\right]\left[B_{1}^{\prime}\right]+\left[B_{3}^{\prime}\right]^{T}\left[E^{\prime}\right]\left[B_{2}^{\prime}\right]+\left[B_{3}^{\prime}\right]^{T}\left[F^{\prime}\right]\left[B_{3}^{\prime}\right]
\end{array}\right]|J| w_{i} w_{j} \\
& {[K]_{e m}=b_{m s} \int\left[\begin{array}{l}
{\left[B_{1 m}\right]^{T}\left[A_{m}\right]\left[B_{1 m}\right]+\left[B_{1 m}\right]^{T}\left[D_{m}\right]\left[B_{3 m}\right]+\left[B_{2 m}\right]^{T}\left[D_{m}\right]\left[B_{2 m}\right]+\left[B_{2 m}\right]^{T}\left[F_{m}\right]\left[B_{4 m}\right]+} \\
+\left[B_{3 m}\right]^{T}\left[D_{m}\right]\left[B_{1 m}\right]+\left[B_{3 m}\right]^{T}\left[F_{m}\right]\left[B_{3 m}\right]+\left[B_{4 m}\right]^{T}\left[F_{m}\right]\left[B_{2 m}\right]+\left[B_{4 m}\right]^{T}\left[H_{m}\right]\left[B_{4 m}\right]+ \\
\left.+\left[B_{1 m}\right]^{T}\left[A_{m}{ }^{\prime}\right]\left[B_{1 m}{ }^{\prime}\right]+\left[B_{1 m}\right]^{T}\right]^{T}\left[D_{m}^{\prime}\right]\left[B_{3 m}{ }^{\prime}\right]+\left[B_{2 m}\right]^{T}\left[D_{m}{ }^{\prime}\right]\left[B_{2 m}{ }^{\prime}\right]+ \\
+\left[B_{3 m}^{\prime}\right]^{T}\left[D_{m}^{\prime}\right]\left[B_{1 m}^{\prime}\right]+\left[B_{3 m}^{\prime}\right]^{T}\left[F_{m}^{\prime}\right]\left[B_{3 m}^{\prime}\right]
\end{array}\right] d l_{e m}} \\
& =b_{m s} \sum_{i=1}^{2}\left[\begin{array}{l}
{\left[B_{1 m}\right]^{T}\left[A_{m}\right]\left[B_{1 m}\right]+\left[B_{1 m}\right]^{T}\left[D_{m}\right]\left[B_{3 m}\right]+\left[B_{2 m}\right]^{T}\left[D_{m}\right]\left[B_{2 m}\right]+\left[B_{2 m}\right]^{T}\left[F_{m}\right]\left[B_{4 m}\right]+} \\
+\left[B_{3 m}\right]^{T}\left[D_{m}\right]\left[B_{1 m}\right]+\left[B_{3 m}\right]^{T}\left[F_{m}\right]\left[B_{3 m}\right]+\left[B_{4 m}\right]^{T}\left[F_{m}\right]\left[B_{2 m}\right]+\left[B_{4 m}\right]^{T}\left[H_{m}\right]\left[B_{4 m}\right]+ \\
\left.+\left[B_{1 m}\right]^{T}\left[A_{m}^{\prime}\right]\left[B_{1 m}^{\prime}\right]+\left[B_{1 m}^{\prime}\right]^{T}\left[D_{m}^{\prime}\right]\left[B_{3 m^{\prime}}\right]^{\prime}\right]+\left[B_{2 m}^{\prime}\right]^{T}\left[D_{m}^{\prime}\right]\left[B_{2 m}{ }^{\prime}\right]+ \\
+\left[B_{3 m}^{\prime}\right]^{T}\left[D_{m}^{\prime}\right]\left[B_{1 m}^{\prime}\right]+\left[B_{3 m}^{\prime}\right]^{T}\left[F_{m}^{\prime}\right]\left[B_{3 m}^{\prime}\right]
\end{array}\right]\left|J_{m}\right| w_{i},
\end{aligned}
$$

with

$$
\begin{aligned}
& m=x, y, \\
& (A, B, D, E, F, H, G)=\int_{-h / 2}^{h / 2}\left[D_{m}\right]\left(1, z, z^{2}, z^{3}, z^{4}, z^{5}, z^{6}\right) d z \\
& \left(A^{\prime}, B^{\prime}, D^{\prime}, E^{\prime}, F^{\prime}\right)=\int_{-h / 2}^{h / 2}\left[D_{s}\right]\left(1, z, z^{2}\right) d z .
\end{aligned}
$$

$|J|$ is det Jacobian matrix

$$
[J]=\left[\begin{array}{cccc}
\frac{\partial N_{1}}{\partial r} & \frac{\partial N_{2}}{\partial r} & \frac{\partial N_{3}}{\partial r} & \frac{\partial N_{4}}{\partial r} \\
\frac{\partial N_{1}}{\partial s} & \frac{\partial N_{2}}{\partial s} & \frac{\partial N_{3}}{\partial s} & \frac{\partial N_{4}}{\partial s}
\end{array}\right]\left[\begin{array}{ll}
x_{1} & y_{1} \\
x_{2} & y_{2} \\
x_{3} & y_{3} \\
x_{4} & y_{4}
\end{array}\right]
$$

and $w_{i}=w_{j}=1$ are Gauss weights for two Gauss points (4 node plate element) and Gauss points $= \pm \frac{1}{\sqrt{3}}$.

$\left|J_{m}\right|$ is det Jacobian matrix

$$
\begin{array}{ll}
{\left[J_{x s}\right]=\left[\begin{array}{ll}
\frac{\partial N_{1}}{\partial r} & \frac{\partial N_{2}}{\partial r}
\end{array}\right]\left[\begin{array}{l}
x_{1} \\
x_{2}
\end{array}\right] \text { for } x \text {-stiffener }} \\
{\left[J_{y s}\right]=\left[\begin{array}{ll}
\frac{\partial N_{1}}{\partial s} & \frac{\partial N_{2}}{\partial s}
\end{array}\right]\left[\begin{array}{l}
y_{1} \\
y_{2}
\end{array}\right] \quad \text { for } y \text {-stiffener }}
\end{array}
$$


and $w_{i}=1$ is Gauss weight for two Gauss points ( 2 node beam element) and Gauss points $= \pm \frac{1}{\sqrt{3}}$.

The kinetic energy of the stiffened plate is computed by

$$
\begin{aligned}
T & =\frac{1}{2} \sum_{N_{p}} \int_{V_{e}}\{\dot{u}\}^{T} \rho\{\dot{u}\} d V_{e}+\frac{1}{2} \sum_{N_{x s} \int_{V_{e x}}}\{\dot{u}\}_{x} \rho_{x}\{\dot{u}\}_{x} d V_{e x}+\frac{1}{2} \sum_{N_{y s} V_{e y}}\{\dot{u}\}_{y} \rho_{y}\{\dot{u}\}_{y} d V_{e y} \\
& =\frac{1}{2} \sum_{N_{p}}\{\dot{q}\}_{e}^{T}\left[M_{p}\right]_{e}\{\dot{q}\}_{e}+\frac{1}{2} \sum_{N_{x s}}\{\dot{q}\}_{e}^{T}\left[M_{x}\right]_{e}\{\dot{q}\}_{e}+\frac{1}{2} \sum_{N_{y s}}\{\dot{q}\}_{e}^{T}\left[M_{y}\right]_{e}\{\dot{q}\}_{e},
\end{aligned}
$$

where

$$
\begin{aligned}
& {\left[M_{p}\right]_{e}=\int_{V_{e}}[N]^{T}[\rho][N] d V_{e}=\int_{-h / 2}^{h / 2}\left(\sum_{i=1}^{2} \sum_{j=1}^{2}[N]^{T}[\rho][N]|J| w_{i} w_{j}\right) d z,} \\
& {\left[M_{x}\right]_{e}=T_{x}^{T}\left(b_{x s} \int_{l_{e x}}\left[N_{x}\right]^{T}\left[\rho_{x}\right]\left[N_{x}\right] d l_{e x}\right) T_{x}=T_{x}^{T}\left(b_{x s} \int_{-h_{x s} / 2}^{h_{x s} / 2}\left(\sum_{i=1}^{2}\left[N_{x}\right]^{T}\left[\rho_{x}\right]\left[N_{x}\right]\left|J_{x s}\right| w_{i}\right) d z\right) T_{x},} \\
& {\left[M_{y}\right]_{e}=T_{y}^{T}\left(b_{y s} \int_{l_{e y}}\left[N_{y}\right]^{T}\left[\rho_{y}\right]\left[N_{y}\right] d l_{e y}\right) T_{y}=T_{y}^{T}\left(b_{y s} \int_{-h_{x s} / 2}^{h_{x s} / 2}\left(\sum_{j=1}^{2}\left[N_{y}\right]^{T}\left[\rho_{y}\right]\left[N_{y}\right]\left|J_{y s}\right| w_{j}\right) d z\right) T_{y} .}
\end{aligned}
$$

In this paper, we apply the Hamilton's principle to find the weak form of the problem (the damping of plate is neglected). The principle is started that

$$
\int_{t_{1}}^{t_{2}}(\delta W+\delta T-\delta U) d t=0,
$$

where $W$ is the work done by external forces on the stiffened plate.

So that Eq. (56) leads to

$$
\left([M]+\left[M_{s}\right]\right)\{\ddot{q}\}+\left([K]+\left[K_{s}\right]\right)\{q\}=\{F\} .
$$
be solved

To obtain natural frequency $(\{F\}=\{0\})$ the following eigenvalue equation must

$$
\left\{\left([K]+\left[K_{s}\right]\right)-\omega^{2}\left([M]+\left[M_{s}\right]\right)\right\}\left\{q_{0}\right\}=\{0\},
$$

where $\omega,\left\{q_{0}\right\}$ - natural frequency and modal shapes.

Integrals in Eqs. (46)-(47) and (55) will be computed by Gauss quadrature [11]. The program for solving Eq. (58) was coded in Matlab. 


\section{NUMERICAL RESULTS}

\subsection{Comparison study}

3.1.1. Free vibration of a simply supported homogeneous rectangular stiffened plate with two stiffeners

A fully simply supported square plate having two centrally placed stiffener has been analyzed by L. X. Peng [12]. The plate and stiffener were made of the same material, with Young's modulus $3.10^{7} \mathrm{~Pa}$, density $2820 \mathrm{~kg} / \mathrm{m}^{3}$, and Poisson's ratio 0.3 (Fig. 4). The first five natural frequencies of this stiffened plate were calculated by using the present theory. Tab. 1 shows calculation results compared with those by mesh-free method of Peng. A good agreement can be seen in this table.

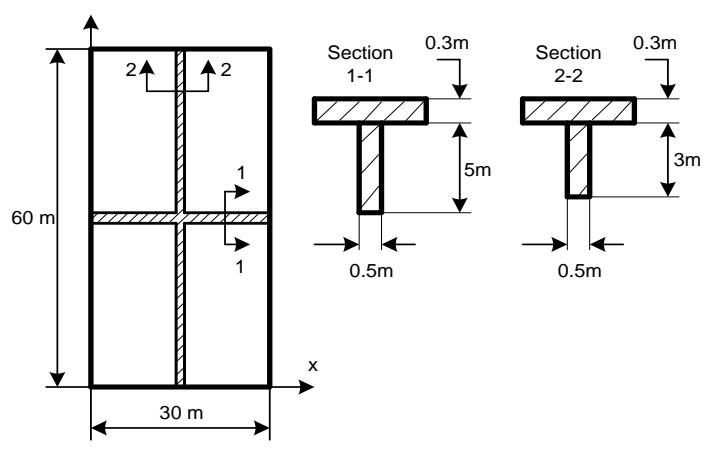

Fig. 4. The stiffened rectangular plate with two stiffeners

Table 1. Comparisons of frequencies for the simply supported homogeneous stiffened plate with two stiffeners

\begin{tabular}{|l|c|c|c|c|c|}
\hline \multirow{2}{*}{} & \multicolumn{5}{|c|}{ Natural frequency (Hz) } \\
\cline { 2 - 6 } & 1 & 2 & 3 & 4 & 5 \\
\hline Present & 0.0821 & 0.0860 & 0.1043 & 0.1063 & 0.1324 \\
\hline L. X. Peng et al. [12] & 0.0816 & 0.0856 & 0.10003 & 0.1028 & 0.1311 \\
\hline
\end{tabular}

\subsubsection{A free vibration of square FG sandwich plate}

A fully simply supported square $\mathrm{FG}$ sandwich plate $\mathrm{Al} / \mathrm{Al}_{2} \mathrm{O}_{3}$ (type 1) with $a=b$ and $2 h$ is thickness, $a /(2 h)=10$. The material properties, as given in L. Hadji [11] are $E_{m}=70 \mathrm{GPa}, v_{m}=0.3, \rho_{m}=2707 \mathrm{~kg} / \mathrm{m}^{3}$ for $\mathrm{Al} ; E_{c}=380 \mathrm{GPa}, v_{c}=0.3, \rho_{c}=3800$ $\mathrm{kg} / \mathrm{m}^{3}$ for $\mathrm{Al}_{2} \mathrm{O}_{3}$; Thickness relation is denoted as the top layer thickness - the core thickness - the bottom thickness $=1-1-1$. The dimensionless frequencies $\omega=\left(\omega a^{2} /(2 h)\right) \sqrt{\rho_{0} / E_{0}}$ (where $\rho_{0}=1 \mathrm{~kg} / \mathrm{m}^{3}, E_{0}=1 \mathrm{GPa}$ ) obtained by the present paper are compared with the first-order shear deformation plate theory (FSDT) (analytical method), the third-order shear deformation plate theory (TSDT) (analytical method), and the four-variable refined 
plate theory [11] (analytical method) in Tab. 2. This comparison once again shows clearly that good agreements are obtained.

Table 2. Comparisons of the dimensionless frequency $\omega$ for simply support FG sandwich plate

\begin{tabular}{|c|c|c|c|c|}
\hline \multirow{2}{*}{$n$} & \multicolumn{4}{|c|}{$\omega$ (thickness relation =1-1-1) } \\
\cline { 2 - 5 } & Present & FSDT [11] & TSDT [11] & Refined plate theory [11] \\
\hline 0 & 1.856207 & 1.82442 & 1.82445 & 1.82445 \\
\hline 0.5 & 1.540301 & 1.51695 & 1.51922 & 1.51921 \\
\hline
\end{tabular}

Table 3. Dimensionless frequency of stiffened FG sandwich plate with one stiffener $\left(a / b=1, b_{s}=a / 50, h_{s}=10 h\right.$, thickness relation $\left.=1: 8: 1\right)$

\begin{tabular}{|c|c|c|c|c|c|}
\hline \multirow{3}{*}{$a /(2 h)$} & \multicolumn{5}{|c|}{ Dimensionless frequency $(\omega)$} \\
\hline & \multirow{2}{*}{$\begin{array}{c}\begin{array}{c}\text { Boundanry } \\
\text { condition }\end{array} \\
n\end{array}$} & \multicolumn{2}{|c|}{ SSSS } & \multicolumn{2}{|c|}{ CCCC } \\
\hline & & First type & Second type & First type & Second type \\
\hline \multirow{5}{*}{40} & & 3.8447 & 5.3852 & 6.6365 & 8.2091 \\
\hline & 0.5 & 3.6780 & 2.7504 & 6.3388 & 4.1175 \\
\hline & 1 & 3.5991 & 2.6715 & 6.1952 & 3.9998 \\
\hline & 2 & 3.5229 & 2.5920 & 6.0551 & 3.8811 \\
\hline & 10 & 3.4174 & 2.4728 & 5.8590 & 3.7032 \\
\hline \multirow{5}{*}{20} & & 3.3175 & 4.8090 & 5.0946 & 7.4121 \\
\hline & 0.5 & 3.1793 & 2.7206 & 4.8855 & 4.0213 \\
\hline & 1 & 3.1140 & 2.6427 & 4.7859 & 3.9075 \\
\hline & 2 & 3.0512 & 2.5641 & 4.6898 & 3.7929 \\
\hline & 10 & 2.9645 & 2.4464 & 4.5567 & 3.6209 \\
\hline \multirow{5}{*}{10} & & 2.5497 & 3.7773 & 3.6246 & 5.3731 \\
\hline & 0.5 & 2.4421 & 2.1815 & 3.4710 & 3.0847 \\
\hline & 1 & 2.3914 & 2.1400 & 3.3984 & 3.0244 \\
\hline & 2 & 2.3428 & 2.0985 & 3.3288 & 2.9639 \\
\hline & 10 & 2.2763 & 2.0369 & 3.2337 & 2.8739 \\
\hline \multirow{5}{*}{5} & & 0.8770 & 1.9684 & 2.5530 & 3.6931 \\
\hline & 0.5 & 0.8726 & 1.5580 & 2.4382 & 2.0938 \\
\hline & 1 & 0.8704 & 1.5268 & 2.3841 & 2.0525 \\
\hline & 2 & 0.8683 & 1.4956 & 2.3323 & 2.0110 \\
\hline & 10 & 0.8654 & 1.4492 & 2.2621 & 1.9489 \\
\hline
\end{tabular}




\subsection{Free vibration of square sandwich FG plate}

3.2.1. Free vibration of FG plate with one central stiffener

- Effect of boundary condition and side-to-thickness ratio

A stiffened FG sandwich plate $\mathrm{Si}_{3} \mathrm{~N}_{4} / \mathrm{SUS} 304$ with $a$ long, $b$ wide and $2 h$ thick. The material properties, as given in Reddy and Chin [13], are $E_{m}=322.7 \mathrm{GPa}, v_{m}=$ $0.28, \rho_{m}=2370 \mathrm{~kg} / \mathrm{m}^{3}$ for $\mathrm{Si}_{3} \mathrm{~N}_{4} ; E_{c}=207.79 \mathrm{GPa}, v_{c}=0.28, \rho_{c}=8166 \mathrm{~kg} / \mathrm{m}^{3}$ for SUS304, thickness relation $=1: 8: 1$. Tabs. 3 and 4 show the nondimensional natural frequencies of different side-to-thickness ratio and volume fraction exponents with four boundary conditions, viz., all edges simply supported (SSSS), all edges clamped (CCCC), two edges opposite simply supported and two edges opposite clamped (CSCS), and two adjacent edges clamped while the other two edges simply supported (CCSS).

Table 4. Dimensionless frequency of stiffened FG sandwich plate with one stiffener $\left(a / b=1, b_{s}=a / 50, h_{s}=10 h\right.$, thickness relation $\left.=1: 8: 1\right)$

\begin{tabular}{|c|c|c|c|c|c|}
\hline \multirow{3}{*}{$a /(2 h)$} & \multicolumn{5}{|c|}{ Dimensionless frequency $(\omega)$} \\
\hline & \multirow{2}{*}{$\begin{array}{c}\begin{array}{c}\text { Boundary } \\
\text { condition }\end{array} \\
n\end{array}$} & \multicolumn{2}{|c|}{ CSCS } & \multicolumn{2}{|c|}{ CCSS } \\
\hline & & First type & Second type & First type & Second type \\
\hline \multirow{5}{*}{40} & & 4.2496 & 6.3919 & 5.0234 & 6.0410 \\
\hline & 0.5 & 4.0924 & 3.7179 & 4.7525 & 3.0867 \\
\hline & 1 & 4.0177 & 3.6466 & 4.6195 & 3.0023 \\
\hline & 2 & 3.9456 & 3.5750 & 4.4887 & 2.9171 \\
\hline & 10 & 3.8457 & 3.4682 & 4.3042 & 2.7892 \\
\hline \multirow{5}{*}{20} & & 3.6826 & 5.6357 & 4.0892 & 5.5741 \\
\hline & 0.5 & 3.5461 & 3.3235 & 3.9068 & 2.9433 \\
\hline & 1 & 3.4813 & 3.2622 & 3.8194 & 2.8680 \\
\hline & 2 & 3.4189 & 3.2009 & 3.7345 & 2.7917 \\
\hline & 10 & 3.3328 & 3.1100 & 3.6164 & 2.6768 \\
\hline \multirow{5}{*}{10} & & 2.8807 & 4.4068 & 2.9509 & 4.3743 \\
\hline & 0.5 & 2.7681 & 2.6098 & 2.8336 & 2.4699 \\
\hline & 1 & 2.7146 & 2.5619 & 2.7771 & 2.4171 \\
\hline & 2 & 2.6632 & 2.5140 & 2.7224 & 2.3638 \\
\hline & 10 & 2.5928 & 2.4432 & 2.6467 & 2.2836 \\
\hline \multirow{5}{*}{5} & & 0.8773 & 1.9696 & 1.5559 & 3.0524 \\
\hline & 0.5 & 0.8728 & 1.8426 & 1.5500 & 1.7762 \\
\hline & 1 & 0.8707 & 1.8068 & 1.5467 & 1.7400 \\
\hline & 2 & 0.8685 & 1.7709 & 1.5433 & 1.7036 \\
\hline & 10 & 0.8656 & 1.7172 & 1.5379 & 1.6492 \\
\hline
\end{tabular}


Remark: The result shows that the natural fundamental frequencies decrease when the power volume index increases. That as $n$ decreases, the ceramic in plate decreases, and so that the rigidity of the plate decreases. In the same conditions (power volume index, side-to-thickness ration), the frequencies are highest for CCCC Stiffened FG sandwich plates followed by CSCS, CCSS, and SSSS stiffened sandwich plate while SSSS stiffened FG sandwich plate has the lowest value of frequencies. This is due to the higher number of constraints introduced in CCCC stiffened plate compared to that of SSSS, CCSS and CSCS plates increasing the stiffness of the plate. The frequencies are to be decreasing with the increasing of the plate's thickness, which because the mass of plates is increased much more than the stiffness.

First four mode shape of stiffened FG sandwich plate (type 1) shows in Fig. 5.

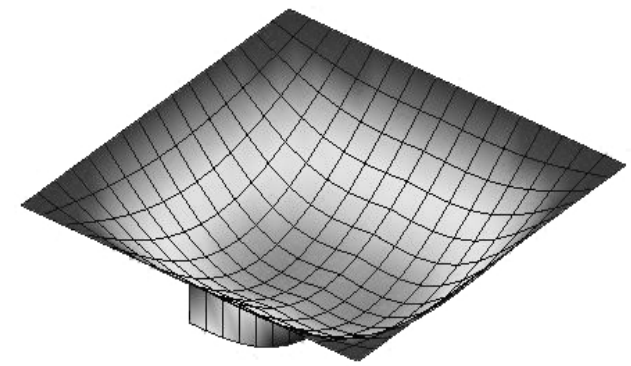

(a) Mode shape $1-2048.508 \mathrm{~Hz}$

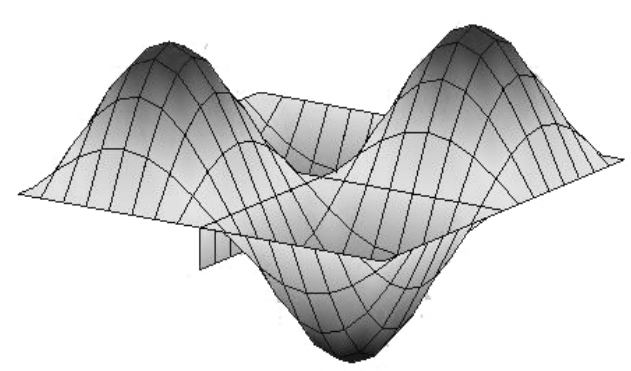

(c) Mode shape 3 - $3936.380 \mathrm{~Hz}$

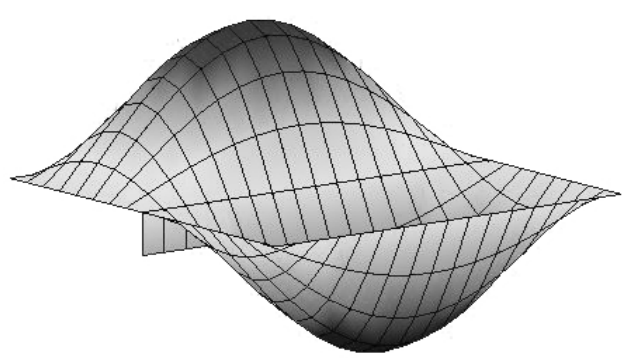

(b) Mode shape 2 - $2621.489 \mathrm{~Hz}$

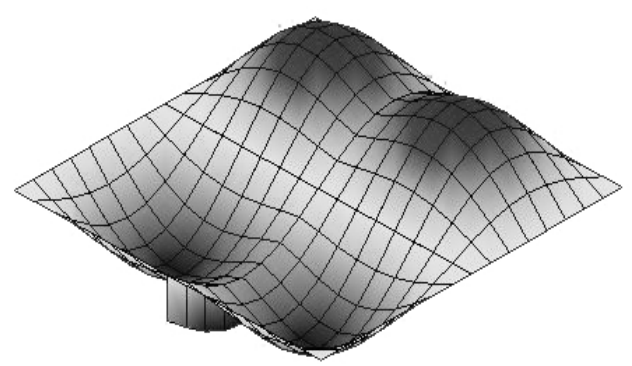

(d) Mode shape 4 - $4838.207 \mathrm{~Hz}$

Fig. 5. Four first linear mode shape of simply support $\mathrm{Si}_{3} \mathrm{~N}_{4} / \mathrm{SUS} 304$ rectangular plate with one stiffener (type $1, a / b=1, a /(2 h)=10, n=0.5, a / b_{s}=50, h_{s}=10 h$, thickness relation $=1-8-1)$

\section{- Effect of core's thickness}

Next, we study the effect of core's thickness for above stiffened plate. Fig. 6 presents the non-dimensionless frequencies with different values of the substrate-to-face sheet thickness ratio $h_{1} / h$ and with different values of the volume fraction index, $n=0$ (ceramic rich), $n=0.5$ (FGM), and $n=10$ (metal rich).

Remark: The results from Fig. 6 show that the frequencies are to be increasing with the increasing of the core's thickness of stiffened plate for type 1, but the frequencies of type 2 
to be decreasing, which because the core's thickness is increased then the ceramic in type 1 is richer than that in type 2 , so that stiffened plate of type 1 becomes stiffener than that of type 2 .

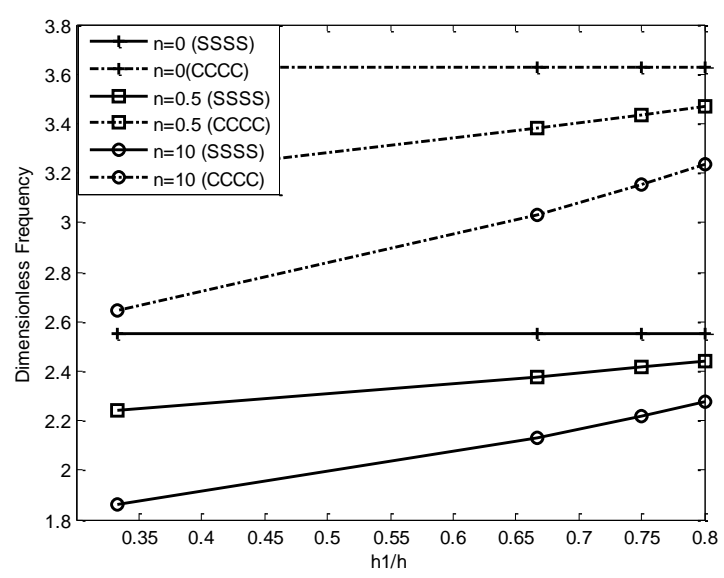

(a) Type 1

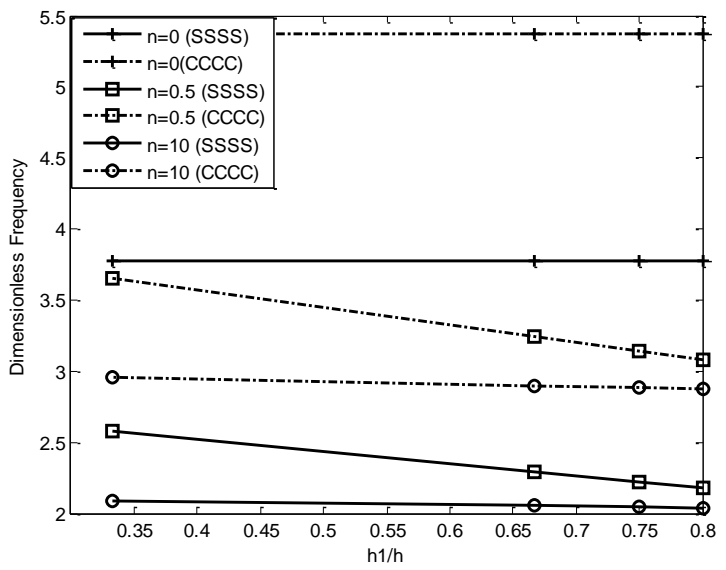

(b) Type 2

Fig. 6. Frequencies vary to $h_{1} / h\left(b_{s}=a / 50, h_{s}=10 h, a / b=1, a /(2 h)=10\right)$

- Effect of stiffener's position

We study the effect of stiffener's position by varying the ratio $x / a$, where $x$ is dimension from one edge of the plate and showed in Fig. 7.



Fig. 7. The rectangular plate with one stiffener

Fig. 8 show dimensionless frequencies results for two types of the plate with two boundary conditions. The results confirm that when the stiffener is closer to center of the plate, the stiffness of the plate becomes higher, so that the corresponding frequencies are higher.

First four mode shapes of simply support (SSSS) stiffened sandwich FGM plate (type 1) for $x / a=0.25$ are shown in Fig. 9 




(a) Type 1

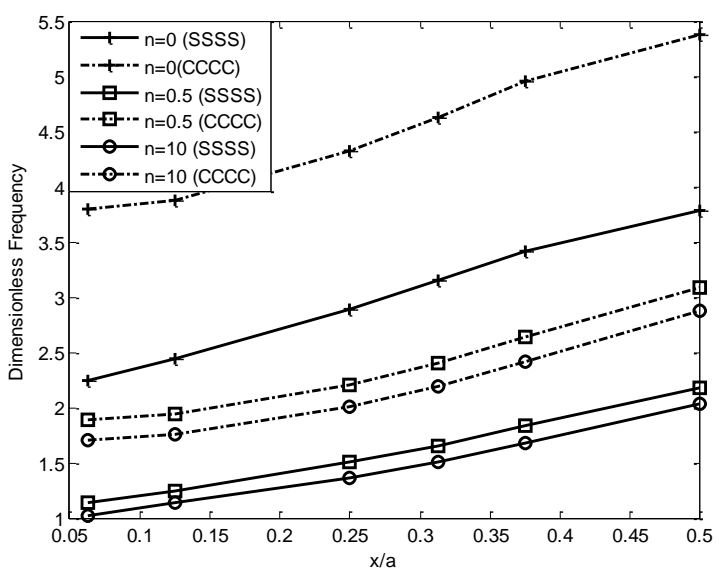

(b) Type 2

Fig. 8. Effects of stiffener's position on frequencies $\left(b_{s}=a / 50, h_{s}=10 h, a / b=1, a /(2 h)=10\right)$



(a) Mode shape $1-1895.171 \mathrm{~Hz}$

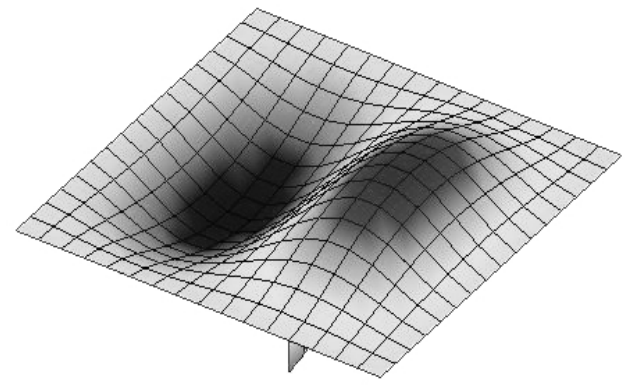

(c) Mode shape 3 - $3216.887 \mathrm{~Hz}$

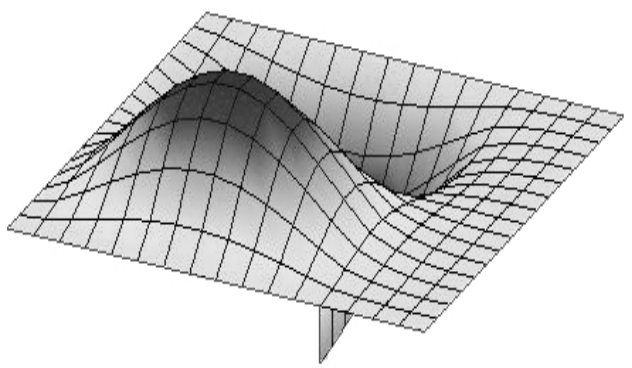

(b) mode shape $2-2608.889 \mathrm{~Hz}$

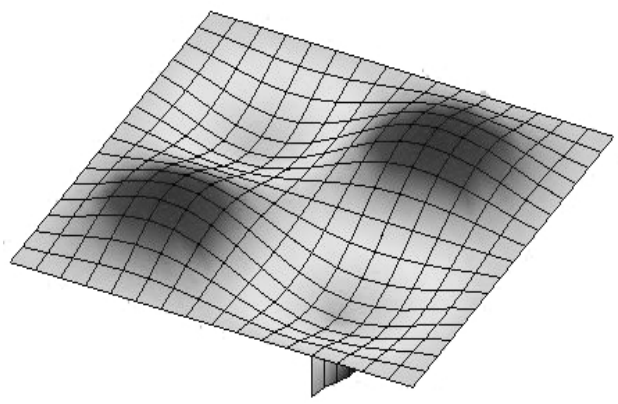

(d) Mode shape 4 - $4394.096 \mathrm{~Hz}$

Fig. 9. First four linear mode shape of full clamp $\mathrm{Si}_{3} \mathrm{~N}_{4} / \mathrm{SUS} 304$ rectangular plate with one stiffener (type $1, a / b=1, a /(2 h)=10, n=0.5, a / b_{s}=50, h_{s}=10 h$, thickness relation $=1: 8: 1$ ) 


\section{- Effect of stiffener's depth}

Next, free vibration analysis of stiffened sandwich plate is carried out for the difference of stiffener's depth. The results from Fig. 10 show that when the depth of stiffener increases, the frequency of the stiffened plate increases.



(a) Type 1

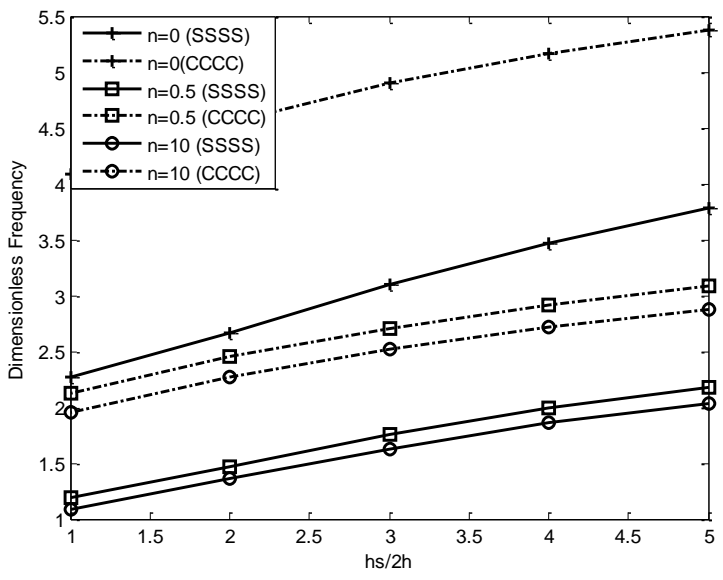

(b) Type 2

Fig. 10. Frequencies vary to $h_{s} / 2 h\left(\right.$ type $\left.1, b_{s}=a / 50, h_{s}=10 h, a / b=1, a /(2 h)=10\right)$

\section{- Effect of stiffener's width}

We study the effect of stiffener's width on the frequencies of the plate. The results of frequencies depending on value of the width are show on Fig. 11. We can see that, when the width increases, the frequency increases for both two boundary conditions and for both two types plate.

\subsubsection{Free vibration of FGM plate with two stiffeners}

Now, we study the free vibration for sandwich plate with two stiffeners, which have the same width and depth. The results are showed in Tabs. 5 and 6.

Remark: The results of plate with two stiffeners have the same signs as that of plate with one stiffener. The natural fundamental frequencies decrease when the power volume index increases. In the same conditions (power volume index, side-tothickness ration), the frequencies are highest for CCCC Stiffened FG sandwich plates followed by CSCS, CCSS, and SSSS stiffened sandwich plate while SSSS stiffened FG sandwich plate has the lowest frequencies. This is due to the higher number of constraints introduced in CCCC stiffened plate compared to that of SSSS, CCSS CSCS plates that increases the stiffness of the plate.

First four mode shape of stiffened FG sandwich plate (type 1) are shows in Fig. 12 




(a) Type 1

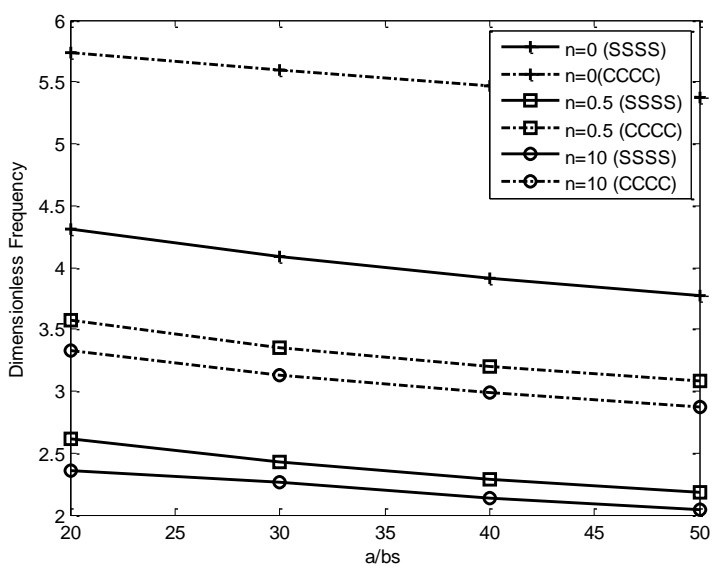

(b) Type 2

Fig. 11. Frequencies vary to $a / b_{s}$ (type $\left.1, b_{s}=a / 50, h_{s}=10 h, a / b=1, a /(2 h)=10\right)$

Table 5. Dimensionless frequency of stiffened FGM sandwich plate with two stiffeners $\left(a / b=1, b_{s}=a / 50, h_{s}=10 h\right.$, thickness relation $=1: 8: 1$

\begin{tabular}{|c|c|c|c|c|c|}
\hline \multirow{3}{*}{$a /(2 h)$} & & \multicolumn{4}{|c|}{ Dimentionless frequency $(\omega)$} \\
\cline { 2 - 6 } & $\begin{array}{l}\text { Boundary } \\
\text { Condition }\end{array}$ & \multicolumn{2}{|c|}{ SSSS } & \multicolumn{2}{c|}{ CCCC } \\
\cline { 2 - 6 } & $n$ & First type & Second type & First type & Second type \\
\hline \multirow{4}{*}{40} & & 4.2478 & 7.0932 & 7.2731 & 11.843 \\
\cline { 2 - 6 } & 0.5 & 4.1417 & 4.3661 & 7.0859 & 6.0555 \\
\cline { 2 - 6 } & 1 & 4.0904 & 4.2718 & 6.9944 & 5.8825 \\
\cline { 2 - 6 } & 2 & 4.040 & 4.1447 & 6.9043 & 5.7082 \\
\hline \multirow{4}{*}{20} & 10 & 3.9699 & 3.9541 & 6.7761 & 5.4468 \\
\cline { 2 - 6 } & & 3.5820 & 6.0887 & 5.3453 & 9.0321 \\
\cline { 2 - 6 } & 0.5 & 3.4919 & 3.8269 & 5.2030 & 5.5521 \\
\cline { 2 - 6 } & 1 & 3.4485 & 3.7651 & 5.1342 & 5.4528 \\
\hline \multirow{4}{*}{10} & 10 & 3.4062 & 3.7030 & 5.0673 & 5.3526 \\
\cline { 2 - 6 } & & 3.3470 & 3.6105 & 4.9737 & 5.2025 \\
\cline { 2 - 6 } & 0.5 & 2.6471 & 4.5195 & 3.5992 & 6.0461 \\
\cline { 2 - 6 } & 1 & 2.5758 & 2.8764 & 3.4935 & 3.8003 \\
\cline { 2 - 6 } & 2 & 2.5416 & 2.8319 & 3.4427 & 3.7384 \\
\hline \multirow{4}{*}{5} & 10 & 2.4683 & 2.7874 & 3.3935 & 3.6765 \\
\cline { 2 - 6 } & & 0.8770 & 1.9684 & 2.3742 & 3.8663 \\
\cline { 2 - 6 } & 1 & 0.8726 & 1.8574 & 2.2980 & 2.3885 \\
\cline { 2 - 6 } & 2 & 0.8704 & 1.8502 & 2.2614 & 2.3485 \\
\cline { 2 - 6 } & 10 & 0.8683 & 1.8427 & 2.2261 & 2.3084 \\
\hline
\end{tabular}


Table 6. Dimensionless frequency of stiffened FGM sandwich plate with two stiffeners $\left(a / b=1, b_{s}=a / 50, h_{s}=10 h\right.$, thickness relation $\left.=1: 8: 1\right)$

\begin{tabular}{|c|c|c|c|c|c|}
\hline \multirow{3}{*}{$a /(2 h)$} & \multicolumn{5}{|c|}{ Dimensionless frequency $(\omega)$} \\
\hline & \multirow{2}{*}{$\begin{array}{c}\text { Boundary } \\
\text { condition } \\
n\end{array}$} & \multicolumn{2}{|c|}{ CSCS } & \multicolumn{2}{|c|}{ CCSS } \\
\hline & & First type & Second type & First type & Second type \\
\hline \multirow{5}{*}{40} & & 5.7643 & 9.4821 & 5.6178 & 8.9041 \\
\hline & 0.5 & 5.6155 & 5.2859 & 5.4666 & 4.7606 \\
\hline & 1 & 5.5433 & 5.1347 & 5.3929 & 4.6312 \\
\hline & 2 & 5.4725 & 4.9823 & 5.3204 & 4.5002 \\
\hline & 10 & 5.3723 & 4.7538 & 5.2173 & 4.3026 \\
\hline \multirow{5}{*}{20} & & 4.4584 & 7.5528 & 4.3655 & 7.3151 \\
\hline & 0.5 & 4.3419 & 4.6961 & 4.2496 & 4.3954 \\
\hline & 1 & 4.2857 & 4.6160 & 4.1936 & 4.3028 \\
\hline & 2 & 4.2310 & 4.5353 & 4.1390 & 4.2071 \\
\hline & 10 & 4.1545 & 4.4147 & 4.0626 & 4.0584 \\
\hline \multirow{5}{*}{10} & & 3.1250 & 5.2927 & 2.9684 & 5.1027 \\
\hline & 0.5 & 3.0371 & 3.3491 & 2.8925 & 3.2354 \\
\hline & 1 & 2.9948 & 3.2956 & 2.8555 & 3.1828 \\
\hline & 2 & 2.9537 & 3.2422 & 2.8194 & 3.1301 \\
\hline & 10 & 2.8969 & 3.1631 & 2.7690 & 3.0517 \\
\hline \multirow{5}{*}{5} & & 0.8773 & 1.9696 & 1.5422 & 3.1982 \\
\hline & 0.5 & 0.8728 & 1.8619 & 1.5360 & 2.1050 \\
\hline & 1 & 0.8707 & 1.8550 & 1.5327 & 2.0702 \\
\hline & 2 & 0.8685 & 1.8477 & 1.5293 & 2.0352 \\
\hline & 10 & 0.8656 & 1.8358 & 1.5242 & 1.9830 \\
\hline
\end{tabular}






(a) Mode shape 1 - $2160.708 \mathrm{~Hz}$



(c) Mode shape 3 - $2621.551 \mathrm{~Hz}$



(b) Mode shape $2-2621.426 \mathrm{~Hz}$

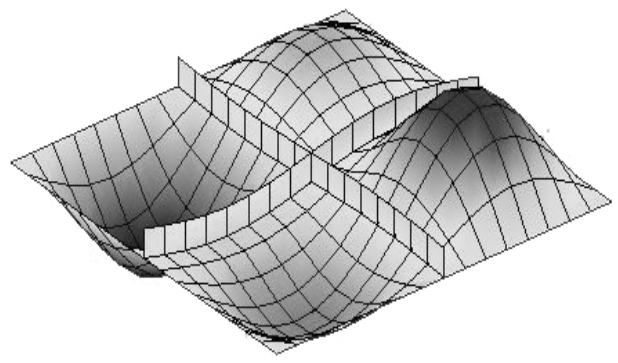

(d) Mode shape 4 - $4837.733 \mathrm{~Hz}$

Fig. 12. First four linear mode shape of simply support $\mathrm{Si}_{3} \mathrm{~N}_{4} / \mathrm{SUS} 304$ sandwich rectangular plate (type 1$)$ with two stiffener $(a / b=1, a / h=20$, $n=0.5, a / b_{s}=50, h_{s}=10 h$, thickness relation $\left.=1-8-1\right)$

\section{CONCLUSIONS}

This paper investigates the free vibration behavior of stiffened FG sandwich plates based on the third order shear deformation and using the finite element method. Two plate configurations, i.e., plate with FG face-sheets and the homogenous core are metal and ceramic, respectively, are considered. The present results are compared to analytical and mesh-free method results given by other researchers to demonstrate a good agreement. Some problems such as the effects of width, depth, position of stiffener, thickness of layers, power volume index, boundary conditions on the natural frequencies of stiffened FG sandwich plate have been investigated. Based on these observations, the method can be recommended for analysis of stiffened FG sandwich plate to predict the frequencies and mode shapes with sufficient accuracy.

\section{REFERENCES}

[1] J. N. Reddy. Analysis of functionally graded plates. International Journal for Numerical Methods in Engineering, 47, (1-3), (2000), pp. 663-684.

[2] S. S. Vel and R. C. Batra. Three-dimensional exact solution for the vibration of functionally graded rectangular plates. Journal of Sound and Vibration, 272, (3), (2004), pp. 703-730. 
[3] T. Q. Bui, T. Van Do, L. H. T. Ton, D. H. Doan, S. Tanaka, D. T. Pham, T.-A. Nguyen-Van, T. Yu, and S. Hirose. On the high temperature mechanical behaviors analysis of heated functionally graded plates using FEM and a new third-order shear deformation plate theory. Composites Part B: Engineering, 92, (2016), pp. 218-241.

[4] D. H. Bich, D. V. Dung, and V. H. Nam. Nonlinear dynamical analysis of eccentrically stiffened functionally graded cylindrical panels. Composite Structures, 94, (8), (2012), pp. 24652473.

[5] D. H. Bich, D. V. Dung, V. H. Nam, and N. T. Phuong. Nonlinear static and dynamic buckling analysis of imperfect eccentrically stiffened functionally graded circular cylindrical thin shells under axial compression. International Journal of Mechanical Sciences, 74, (2013), pp. 190200.

[6] N. D. Duc and P. H. Cong. Nonlinear postbuckling of an eccentrically stiffened thin FGM plate resting on elastic foundations in thermal environments. Thin-Walled Structures, 75, (2014), pp. 103-112.

[7] N. D. Duc and P. H. Cong. Nonlinear postbuckling of symmetric S-FGM plates resting on elastic foundations using higher order shear deformation plate theory in thermal environments. Composite Structures, 100, (2013), pp. 566-574.

[8] A. M. Zenkour. A comprehensive analysis of functionally graded sandwich plates: Part 1Deflection and stresses. International Journal of Solids and Structures, 42, (18), (2005), pp. 5224 5242.

[9] M. M. Alipour and M. Shariyat. An elasticity-equilibrium-based zigzag theory for axisymmetric bending and stress analysis of the functionally graded circular sandwich plates, using a Maclaurin-type series solution. European Journal of Mechanics-A/Solids, 34, (2012), pp. 78101.

[10] S. Goswami and W. Becker. A new rectangular finite element formulation based on higher order displacement theory for thick and thin composite and sandwich plates. World Journal of Mechanics, 3, (03), (2013), p. 194.

[11] L. Hadji, H. A. Atmane, A. Tounsi, I. Mechab, and E. A. A. Bedia. Free vibration of functionally graded sandwich plates using four-variable refined plate theory. Applied Mathematics and Mechanics, 32, (7), (2011), pp. 925-942.

[12] L. X. Peng, K. M. Liew, and S. Kitipornchai. Buckling and free vibration analyses of stiffened plates using the FSDT mesh-free method. Journal of Sound and Vibration, 289, (3), (2006), pp. 421-449.

[13] J. N. Reddy and C. D. Chin. Thermomechanical analysis of functionally graded cylinders and plates. Journal of Thermal Stresses, 21, (6), (1998), pp. 593-626. 